\title{
Scaling of F-Actin Network Rheology to Probe Single Filament Elasticity and Dynamics
}

\section{Citation}

Gardel, M. L., J. H. Shin, F. C. MacKintosh, L. Mahadevan, P. A. Matsudaira, and D. A. Weitz. 2004. "Scaling of F-Actin Network Rheology to Probe Single Filament Elasticity and Dynamics." Physical Review Letters 93 (18). https://doi.org/10.1103/physrevlett.93.188102.

\section{Permanent link}

http://nrs.harvard.edu/urn-3:HUL.InstRepos:41417291

\section{Terms of Use}

This article was downloaded from Harvard University's DASH repository, and is made available under the terms and conditions applicable to Other Posted Material, as set forth at http:// nrs.harvard.edu/urn-3:HUL.InstRepos:dash.current.terms-of-use\#LAA

\section{Share Your Story}

The Harvard community has made this article openly available.

Please share how this access benefits you. Submit a story.

Accessibility 


\title{
Scaling of F-Actin Network Rheology to Probe Single Filament Elasticity and Dynamics
}

\author{
M. L. Gardel,,${ }^{1,2}$ J. H. Shin, ${ }^{3,4}$ F. C. MacKintosh,${ }^{5}$ L. Mahadevan, ${ }^{2}$ P. A. Matsudaira, ${ }^{4}$ and D. A. Weitz ${ }^{1,2}$ \\ ${ }^{1}$ Department of Physics, Harvard University, Cambridge, Massachusetts 02138, USA \\ ${ }^{2}$ Division of Engineering and Applied Sciences, Harvard University, Cambridge, Massachusetts 02138, USA \\ ${ }^{3}$ Department of Mechanical Engineering, M.I.T., Cambridge Massachusetts 02142, USA \\ ${ }^{4}$ Whitehead Institute for Biomedical Research, Department of Biology and Division of Biological Engineering, MIT, \\ Cambridge, Massachusetts 02142, USA \\ ${ }^{5}$ Division of Physics and Astronomy, Vrije Universiteit, Amsterdam, The Netherlands
}

(Received 30 December 2003; published 29 October 2004)

\begin{abstract}
The linear and nonlinear viscoelastic response of networks of cross-linked and bundled cytoskeletal filaments demonstrates remarkable scaling with both frequency and applied prestress, which helps elucidate the origins of the viscoelasticity. The frequency dependence of the shear modulus reflects the underlying single-filament relaxation dynamics for $0.1-10 \mathrm{rad} / \mathrm{sec}$. Moreover, the nonlinear strain stiffening of such networks exhibits a universal form as a function of prestress; this is quantitatively explained by the full force-extension relation of single semiflexible filaments.
\end{abstract}

Actin is a protein found in abundance in all eukaryotic cells; it can polymerize to form semiflexible filaments (F actin). Reconstituted actin filaments are an excellent model for the study of the properties of entangled semiflexible polymer solutions [1-4]. In vivo, a myriad of actin binding proteins can both cross-link and bundle actin filaments to create rigid actin networks that provide an essential mechanical component in living cells; these networks are critical in cell morphology, mechanoprotection, and motility [5]. The elasticity of these networks depends crucially on the semiflexibility of individual filaments, as well as the interactions between filaments due to cross-links or bundles [1,6-9]. Knowledge of the underlying origin of the elasticity of such cross-linked networks is essential to determine the precise role of the behavior of the cytoskeleton in cellular functions. The elasticity of entangled solutions of actin filaments, in the absence of cross-links, is entropic in origin [3,4]. As the degree of cross-linking or bundling increases, the role of entropy should decrease as enthalpic effects become more important [8,9]. However, the relative contribution of enthalpic effects, and the contribution of the entropic fluctuations of the actin filaments that make up in vivo networks remain unclear. Thus, the underlying mechanisms of the mechanical response of cross-linked actin networks remain largely unknown.

In this Letter, we demonstrate that the macroscopic mechanical response of F-actin networks can be explained quantitatively in terms of single-filament elasticity and dynamics, over a large range of filament and cross-linker concentrations. We show that the linear viscoelasticity of cross-linked networks of $\mathrm{F}$ actin can be scaled onto a single master curve as a function of frequency, despite orders of magnitude variation in the elastic moduli. This scaling reveals that the relaxation dynamics of single filaments is the dominant mechanism of dissipation in these cross-linked networks, even at low frequencies. We also probe the nonlinear elastic response of the network by prestressing the networks with an external steady shear stress. We observe a dramatic stress stiffening of the elastic response for cross-linked networks; this follows a universal form which can be quantitatively explained by the force-extension relation of a single semiflexible polymer. These results demonstrate that the entropic effects of single filaments dominate the full range of mechanical and dynamical properties of F-actin networks.

We use scruin to rigidly cross-link and bundle actin filaments [10]. Found in vivo in the acrosome of the horseshoe-crab sperm, scruin heterodimers bind to adjacent actin monomers on an individual actin filament; cross-links between neighboring filaments are mediated through scruin-scruin interactions [11,12]. Scruin has the advantage that its bonds are both noncompliant and irreversible, ensuring that the network response reflects that of the actin filaments alone [13]. We vary the degree of cross-linking and bundling of filaments by altering the relative concentrations of scruin, $c_{S}$, to actin, $c_{A}$. Thus we tune $R=c_{S} / c_{A}$ from zero to one. Solutions of monmeric actin are prepared and stored at $4{ }^{\circ} \mathrm{C}$ in G-buffer [ $2 \mathrm{mM}$ Tris $\mathrm{HCl}, 0.2 \mathrm{mM}$ ATP (adenine triphosphate), $0.2 \mathrm{mM} \mathrm{CaCl} 2,0.2 \mathrm{mM}$ DTT (dithiothreitol), $0.005 \% \mathrm{NaN}_{3}, p \mathrm{H} \mathrm{8.0]}$ for up to $10 \mathrm{~d}$. Samples are prepared by gently mixing monomeric actin, scruin, and $10 x$ F-buffer $(20 \mathrm{mM}$ Tris $\mathrm{HCl}, 20 \mathrm{mM} \mathrm{MgCl} 2,1 \mathrm{M} \mathrm{KCl}$,

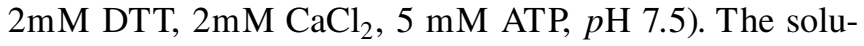
tion is loaded within 1 min of mixing into a stresscontrolled rheometer with $40 \mathrm{~mm}$ parallel plate geometry and $160 \mu \mathrm{m}$ plate separation; the sample volume is $\sim 250 \mu \mathrm{L}$. We confirm that the results are independent of geometry. We use a solvent trap to prevent drying, and equilibrate the sample for $1 \mathrm{~h}$ at $25^{\circ} \mathrm{C}$. We measure the 
frequency-dependent linear elastic, $G^{\prime}(\omega)$, and viscous, $G^{\prime \prime}(\omega)$, moduli using a maximum strain of $\gamma \sim 0.03$.

The mechanical response of all the networks over a large range of $c_{A}$ and $R$ is dominated by a nearly frequency independent elastic modulus which defines the elastic plateau modulus $G_{0}$. The magnitude of $G_{0}$ can be varied over 3 orders of magnitude by changing either $c_{A}$ or $R$. For example, $G_{0}$ increases from 0.1 to $300 \mathrm{~Pa}$ when $R$ is increased from 0.001 (squares) to one (circles) for a constant actin concentration, $c_{A}=11.9 \mu \mathrm{M}$, as shown in Fig. 1(a). In contrast, $G^{\prime \prime}(\omega)$ is frequency dependent and does not vary uniformly with $G_{0}$, as shown by the open symbols in Fig. 1(a). In general, as $G_{0}$ increases, $G^{\prime \prime}(\omega)$ becomes more frequency independent and its magnitude relative to $G_{0}$ decreases.

Remarkably, despite these differences, the frequencydependent viscoelastic response of all networks can be scaled onto a single pair of master curves, as shown in Fig. 1(c); this is accomplished by independently scaling both the moduli and the frequency for each data set: the moduli are scaled by a factor $b$, while the frequency is scaled by a factor $a$. The scaled master curves highlight the fact that $G^{\prime}(\omega)$ is nearly frequency independent and dominates the mechanical response over nearly six decades in scaled frequency, $\omega a$. Scaling of the mechanical response is ubiquitous in networks [14], having been observed previously in microrheology measurements of
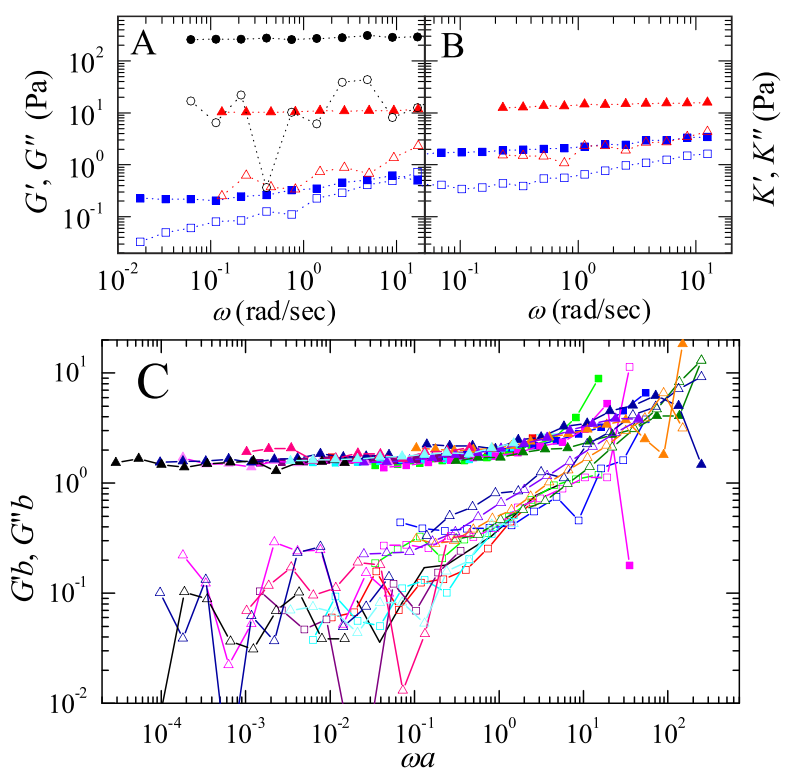

FIG. 1 (color online). $\quad G(\omega)$ (solid symbols) and $G^{\prime \prime}(\omega)$ (open symbols) of composite actin:scruin networks with (a) $c_{A}=$ $11.9 \mu \mathrm{M}, R=0.001$ (squares), $c_{A}=17.85 \mu \mathrm{M}, R=0.16$ (triangles) and $c_{A}=11.9 \mu \mathrm{M}, R=1$ (circles) and (b) $c_{A}=$ $11.9 \mu \mathrm{M}, R=0.13$ with $\sigma_{0}=0 \mathrm{~Pa}$ (squares) $\sigma_{0}=0.9 \mathrm{~Pa}$ (triangles). (c) The master curve of frequency-dependent viscoelasticity composite scruin:actin networks compiled by scaling the bulk rheology of networks varying $0.001<R<$ $1,5<c_{A}<30 \mu \mathrm{M}$ and $0<\sigma_{0}<5 \mathrm{~Pa}$. actin networks [15] and in rheology measurements of weakly attractive colloidal gels [16].

By analogy to the scaling behavior of colloidal gels [16], we assume that the complex viscoelasticity of crosslinked actin networks has two distinct contributions: At low frequencies, it is dominated by a frequencyindependent elastic component, $G^{\prime}(\omega) \sim R^{\beta} c_{A}^{\alpha}$, determined by the network architecture, and, at high frequencies, it is dominated a frequency-dependent component, $G^{\prime}(\omega) \sim G^{\prime \prime}(\omega) \sim c_{A} \omega^{z}$, due to dynamics of individual actin filaments, and linearly dependent on $c_{A}[17,18]$. Thus, the scaling factors can be rewritten as $b \sim$ $c_{A}^{-\alpha} R^{-\beta}$ and $a \sim c_{A}^{(1-\alpha) / z} R^{-\beta / z}$, which implies that $b c_{A} \sim$ $a^{z}$. We do indeed observe such power law scaling over nearly five decades, as shown in Fig. 2, confirming that this model correctly accounts for the scaling and captures the underlying origin of the network viscoelasticity. Moreover, the power law we observe is consistent with $z=3 / 4$, indicated by the solid line Fig. 2 . Despite the fact that our measurements are restricted to low frequencies, the scaling behavior highlights high-frequency contributions. Thus, this is direct evidence, using bulk rheology, that the high-frequency response is $\sim \omega^{3 / 4}$, and reflects the high-frequency behavior of individual filaments $[17,18]$. Unlike the colloidal gels, the frequencydependent elasticity of cross-linked actin networks is determined by two distinct contributions that arise from a single component, individual actin filaments.

These results suggest that thermal fluctuations of actin filaments dominate the frequency-dependent mechanical response of the network. The entropic spring constant of a thermally fluctuating, semiflexible filament arises from the reduction of the fluctuations in end-to-end distance of the filament as it is extended from its thermally contracted length to its full contour length. This entropic spring constant is highly dependent on the length of the filament, which is determined by the number of crosslinks [7]. This is consistent with the large increase in $G_{0}$ of the network observed with an increase in $R$ [Fig. 1(a)].

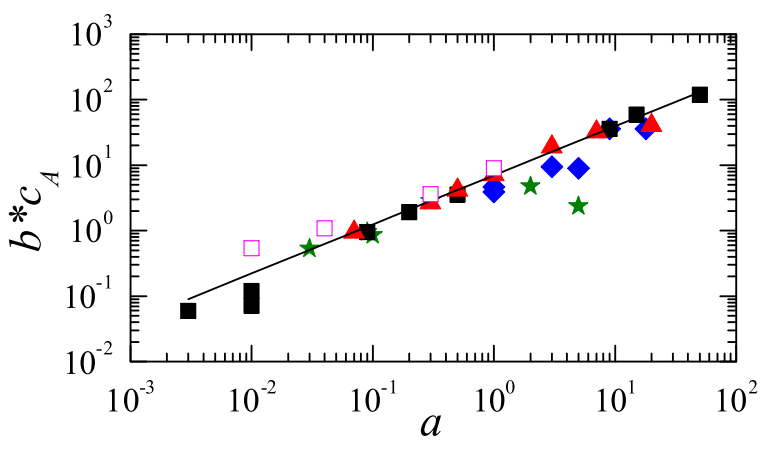

FIG. 2 (color online). The scaling of the scaling parameters as a function of $R$ for $c_{A}=12 \mu \mathrm{M}$ (squares), $c_{A}$ for $R=0.16$ (triangles), $c_{A}$ for $R=0.03$ (diamonds), $c_{A}$ for $R=0.3$ (stars) and prestress for $c_{A}=0.5$ and $R=0.16$ (open squares). The solid line shows a scaling of $a^{3 / 4}$. 
At high extensions, as the end-to-end length approaches the contour length, the requisite force diverges as $F \sim$ $\left|\epsilon-\epsilon_{\max }\right|^{-2}$, where $\epsilon$ is the extension and $\epsilon_{\max }$ is the maximum possible extension $[7,19,20]$. The corresponding network modulus is determined from the stress, $\sim F / \xi^{2}$, where $\xi \sim c_{A}^{-1 / 2}$ is the mesh size. This modulus should diverge with increasing strain, reflecting the divergence in $F$. This suggests that a more sensitive test of the entropic origin of the network elasticity would be to measure the response under an applied prestress. When a steady shear stress, $\sigma_{0}$, is applied, we observe a resultant strain $\gamma_{0}=\sigma_{0} / G_{0}$ that does not vary with time. Upon removal of the shear stress, the strain recovers quickly to zero; thus, the network response is predominately elastic and is completely recoverable. However, as the magnitude of the shear stress increases beyond the linear-response regime, $\gamma_{0}$ approaches a maximum; this reflects a dramatic decrease in the network compliance and directly reflects the corresponding divergence of the elastic modulus.

To explore this dramatic stress stiffening in the nonlinear elastic regime, we measure the differential, or incremental, elastic modulus. We superpose a small amplitude oscillatory stress, $\delta \sigma(\omega) \sim|\delta \sigma| e^{i \omega t}$ on a given $\sigma_{0}$, and measure the oscillatory strain response, $\delta \gamma(\omega) \sim$ $|\delta \gamma| e^{i \omega t}$; when $|\delta \sigma(\omega)| \leq \sigma_{0} / 10$, the oscillatory response is linear for all $\sigma_{0}$. Thus, the complex differential elastic modulus is $K^{*}\left(\omega, \sigma_{0}\right)=\left.[\delta \sigma(\omega) / \delta \gamma(\omega)]\right|_{\sigma_{0}}$. For small values of $\sigma_{0}$, the frequency-dependent differential elastic modulus, $K^{\prime}\left(\omega, \sigma_{0}\right)$, and loss modulus, $K^{\prime \prime}\left(\omega, \sigma_{0}\right)$ are identical to $G^{\prime}(\omega)$ and $G^{\prime \prime}(\omega)$, respectively. As $\sigma_{0}$ is increased above some critical value, $\sigma_{\text {crit }}$, the differential elastic modulus increases dramatically as shown in Fig. 1(b) for a network with $c_{A}=11.9 \mu \mathrm{M}$ and $R=$ $0.13 ; K^{\prime}(\omega)$ increases by nearly a decade as $\sigma_{0}$ in increased from $0 \mathrm{~Pa}$ (solid squares) to $0.9 \mathrm{~Pa}$ (solid triangles). The differential moduli continue to increase as a function of $\sigma_{0}$ until the network catastrophically breaks at a maximum stress, $\sigma_{\max }$. Intriguingly, we can rescale the frequency-dependent response of the prestressed networks onto the same master curve of linear frequencydependent moduli with similar scaling parameters for the zero prestress networks, as shown by the open squares in Fig. 2. The slope of the scaling parameters for the prestressed samples may be somewhat less than $3 / 4$, which is consistent with the dynamics of filaments under static tension $[21,22]$.

To quantify the nonlinear behavior, we measure $K^{\prime}\left(\sigma_{0}\right)$ at a constant frequency, $\omega=0.62 \mathrm{rad} / \mathrm{sec}$, as a function of $\sigma_{0}$. For $c_{A}=29.4 \mu \mathrm{M}$ and $R=0.03, K^{\prime}\left(\sigma_{0}\right)$ is constant for $0<\sigma_{0}<1 \mathrm{~Pa}$ and its magnitude, $9 \mathrm{~Pa}$, is identical to $G_{0}$, as shown by the solid circles in Fig. 3; above $\sigma_{0}=1 \mathrm{~Pa}, K^{\prime}$ increases rapidly, until the network catastrophically breaks at $\sigma_{\max }=14 \mathrm{~Pa}$. We observe qualitatively similar divergences of $K^{\prime}\left(\sigma_{0}\right)$ as $c_{A}$ is decreased, as shown by the solid data points Fig. 3. At low $\sigma_{0}, K^{\prime}$

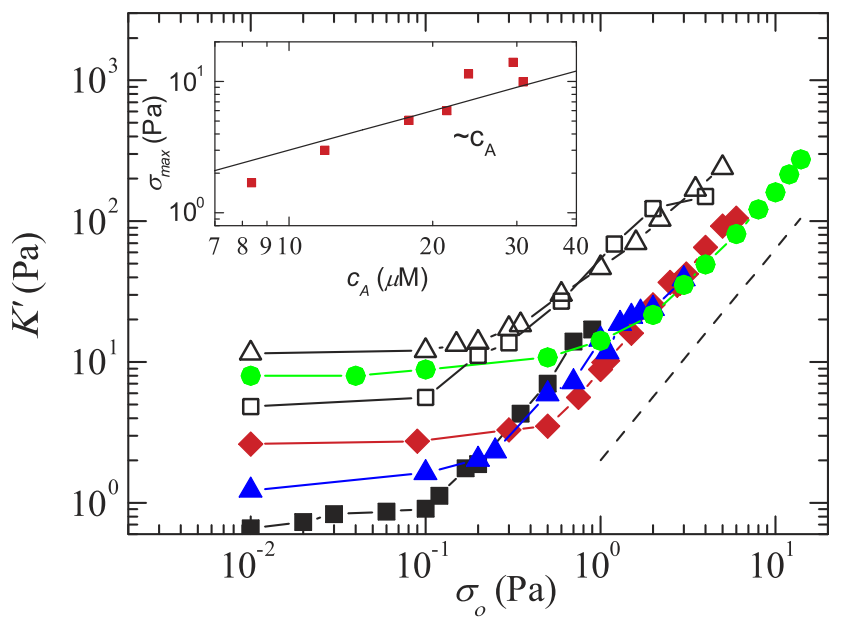

FIG. 3 (color online). $\quad K^{\prime}$ at $0.6 \mathrm{rad} / \mathrm{sec}$ as a function of $\sigma_{0}$ for composite networks: $\left[R=0.03\right.$ with $c_{A}=29.4 \mu \mathrm{M}$ (solid circles), $21.4 \mu \mathrm{M}$ (solid diamonds), $11.9 \mu \mathrm{M}$ (solid triangles), $8.33 \mu \mathrm{M}$ (solid squares) and $R=0.5$ with $c_{A}=7 \mu \mathrm{M}$ (open triangles) and $3 \mu \mathrm{M}$ (open squares)]. The inset shows $\sigma_{\max }$ as a function of $c_{A}$ for $R=0.03$. The solid line shows a scaling $\sim c_{A}$.

decreases with $c_{A}$, reflecting the decrease in $G_{0}$, whereas at high $\sigma_{0}, K^{\prime}$ appears to asymptotically increase to the same values. A similar divergence is seen for $R=0.5$, as illustrated by the open symbols in Fig. 3. In this case, the filaments form thicker bundles [23], resulting in a significant reduction in the critical stress.

To elucidate the mechanisms of this nonlinear elasticity, we examine the behavior of $\sigma_{\max }$. For $R=0.03, \sigma_{\max }$ increases approximately linearly with $c_{A}$, as shown by the solid line in the inset of Fig. 3. This trend is consistent with the behavior expected if the networks break by individual actin-filament rupture. A naive estimate of the breaking stress of a $24-\mu \mathrm{M}$ cross-linked actin network is found by dividing the rupture force of an individual filament, $\sim 300 \mathrm{pN}$ [24], by the area per filament, $\xi^{2} \approx 0.1 \mu \mathrm{m}^{2}$ [25]. However, given the nonlinear forceextension relation for a semiflexible filament in an isotropic network, the stress is effectively concentrated in the small fraction of filaments oriented at $45^{\circ}$ in the shear plane. Accounting for these effects, we find that the maximally stressed filaments are expected to rupture for network stresses of order tens of $\mathrm{Pa}$ [23], consistent with our observations. Furthermore, we observe that, for constant $c_{A}, \sigma_{\max }$ is independent of cross-link density between $0.01 \leq R \leq 0.4$. This further supports our hypothesis that the mechanism of network breakage is by actin-filament rupture rather than stress-induced crosslink unbinding.

The nonlinear mechanical response of the network directly reflects the nonlinear elastic response of single filaments [7]. We observe an increase of $K^{\prime}(\sigma)$ that is consistent with $\sigma^{3 / 2}$, as indicated by the dashed line in Fig. 3; this reflects the nonlinear spring constant, 


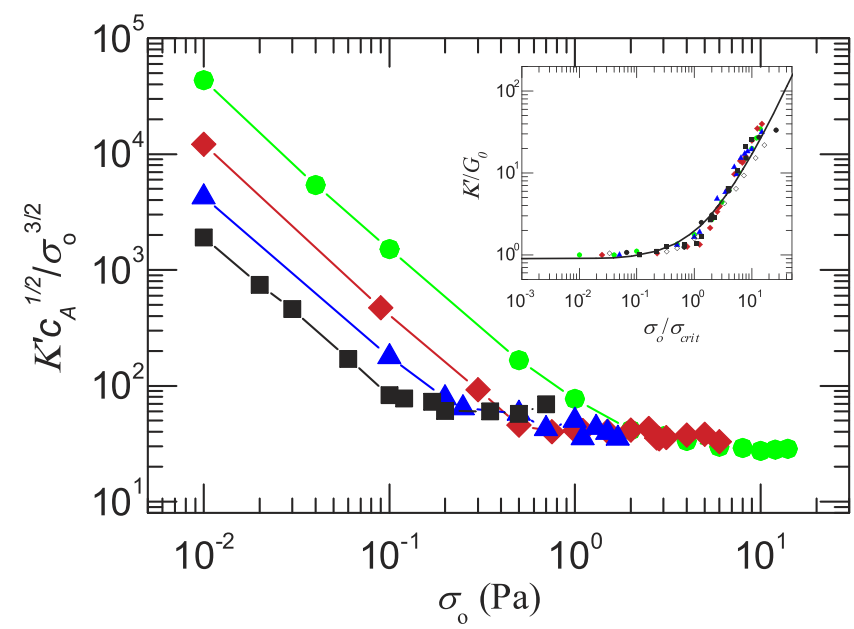

FIG. 4 (color online). $\quad K^{\prime}$ of the $R=0.03$ data shown in Fig. 3 divided by $\sigma_{o}^{3 / 2}$ as a function of $\sigma_{0}$. The inset shows $K^{\prime}$ scaled by $G_{0}$ as a function of $\sigma_{0} / \sigma_{\text {crit }}$ for all the data in Fig. 3. The solid line in the inset indicates the theoretical prediction.

$d F / d \epsilon \sim F^{3 / 2}$, obtained from the divergence of $F$ as the filaments approach full extension. More precisely, $K^{\prime} \sim$ $d \sigma / d \gamma \sim c_{A}^{-1 / 2} \sigma^{3 / 2}$. To test this prediction, we plot $c_{A}^{1 / 2} K^{\prime}\left(\sigma_{0}\right) / \sigma_{0}^{3 / 2}$. It approaches a constant value at high $\sigma_{0}$, as shown in Fig. 4. This is consistent with our prediction.

This universality of the stress stiffening response is highlighted by rescaling the data sets by $G_{0}$ and $\sigma_{\text {crit }}$. The stress stiffening responses for all the cross-linked and bundled networks exhibit identical functional forms, as shown in Fig. 4. Moreover, we can plot the theoretically predicted form for a network of affinely strained, semiflexible polymers directly onto the scaled data; the agreement is very good, as shown by the solid line in the inset of Fig. 4 [23]. This is further confirmation of our hypothesis that the full elastic response of the prestressed networks can be directly understood in terms of the entropic stretching of single filaments.

Scaling the bulk rheology of networks is a powerful means of elucidating microscopic mechanisms of network linear and nonlinear viscoelasticity. However, such scaling of the linear rheology may not be observed in entangled F-actin solutions without physical cross-links, where additional relaxation mechanisms may exist [26]. Furthermore, scaling of the nonlinear elasticity may not occur when the stress stiffening response is due to reorganization of the microstructure or if the network response is nonaffine [27,28]. Moreover, we expect that highly strained networks may begin to show evidence of enthalpic or nonaffine effects under high stress for some concentrations of cross-links or filaments. However, the results here provide convincing evidence for the role of entropic single-filament response over a wide range of network parameters. These highly cross-linked actin net- works are more akin to those found in cytoskeletal networks in vivo, and the results presented here will help elucidate the origin of the elasticity of in vivo networks.

We thank P. A. Janmey, T. C. Lubensky, C. Storm, and $\mathrm{V}$. Trappe for useful discussions. This work was supported by the NSF (DMR-0243715), NSF (J.H. S.) and a Lucent GRPW (M. L. G.).

[1] P. Janmey, S. Hvidt, J. Lamb, and T. Stossel, Nature (London) 345, 89 (1990).

[2] R. Ruddies, W. Goldmann, G. Isenberg, and E. Sackmann, Eur. Biophys. J. 22, 309 (1993).

[3] H. Isambert and A. Maggs, Macromolecules 29, 1036 (1996).

[4] D. C. Morse, Macromolecules 31, 7044 (1998).

[5] H. Lodish, A. Berk, S. Zipursky, P. Matsudaira, D. Baltimore, and J. Darnell, Molecular Cell Biology (W. H. Freeman Co., New York, 1999).

[6] D. H. Wachsstock, W. H. Schwarz, and T. D. Pollard, Biophys. J. 66, 801 (1994).

[7] F. C. MacKintosh, J. Kas, and P. Janmey, Phys. Rev. Lett. 75, 4425 (1995).

[8] K. Kroy and E. Frey, Phys. Rev. Lett. 77, 306 (1996).

[9] R. L. Satcher and C. F. Dewey, Biophys. J. 71, 109 (1996).

[10] L. Tilney, J. Cell Biol. 64, 289 (1975).

[11] M. Schmid, J. Agris, J. Jakana, P. Matsudaira, and W. Chiu, J. Cell Biol. 124, 341 (1994).

[12] M. Sherman, J. Jakana, S. Sun, P. Matsudaira, W. Chiu, and M. Schmid, J. Mol. Biol. 294, 139 (1999).

[13] J. H. Shin, L. Mahadevan, P.T. So, and P. Matsudaira, J. Mol. Biol. 337, 256 (2004).

[14] D. Adolf and J.E. Martin, Macromolecules 23, 3700 (1990).

[15] T. Gisler and D. A. Weitz, Phys. Rev. Lett. 82, 1606 (1999).

[16] V. Trappe and D. Weitz, Phys. Rev. Lett. 85, 449 (2000).

[17] F. Gittes and F. MacKintosh, Phys. Rev. E 58, 1241(R) (1998).

[18] D. C. Morse, Phys. Rev. E 58, 1237(R) (1998).

[19] M. Fixman and J. Kovac, J. Chem. Phys. 58, 1564 (1973).

[20] C. Bustamante, J. F. Marko, E. D. Siggia, and S. Smith, Science 265, 1599 (1994).

[21] R. Granek, J. Phys. II (France) 7, 1761 (1997).

[22] A. Caspi, M. Elbaum, R. Granek, A. Lachish, and D. Zbaida, Phys. Rev. Lett. 80, 1106 (1998).

[23] L. M. P. M. J.H. Shin, M. L. Gardel, and D. Weitz, Proc. Natl. Acad. Sci. U.S.A. 101, 9636 (2004).

[24] Y. Tsuda, H. Yasutake, A. Ishijima, and T. Yanagida, Proc. Nat. Acad. Sci. U.S.A. 93, 12937 (1996).

[25] C. F. Schmidt, M. Baermann, G. Isenberg, and E. Sackmann, Macromolecules 22, 3638 (1989).

[26] M. Pasquali, V. Shankar, and D. Morse, Phys. Rev. E 64, 020802 (2001).

[27] D. Head, A. Levine, and F. C. MacKintosh, Phys. Rev. Lett. 91, 108102 (1) (2003).

[28] J. Wilhelm and E. Frey, Phys. Rev. Lett. 91, 108103 (2003). 